\title{
Beam-helicity asymmetries in double charged pion photoproduction on the proton
}

\author{
S. Strauch
}

Angela Biselli

Fairfield University, abiselli@fairfield.edu

CLAS Collaboration

Follow this and additional works at: https://digitalcommons.fairfield.edu/physics-facultypubs

Copyright American Physical Society

Final Publisher version also available at http://prl.aps.org/abstract/PRL/v95/i16/e162003

\section{Peer Reviewed}

\section{Repository Citation}

Strauch, S.; Biselli, Angela; and CLAS Collaboration, "Beam-helicity asymmetries in double charged pion photoproduction on the proton" (2005). Physics Faculty Publications. 6.

https://digitalcommons.fairfield.edu/physics-facultypubs/6

\section{Published Citation}

S. Strauch et al. [CLAS Collaboration], "Beam-helicity asymmetries in double charged pion photoproduction on the proton", Phys. Rev. Lett. 95, 162003 (2005) DOI: 10.1103/PhysRevLett.95.162003

This item has been accepted for inclusion in DigitalCommons@Fairfield by an authorized administrator of DigitalCommons@Fairfield. It is brought to you by DigitalCommons@Fairfield with permission from the rightsholder(s) and is protected by copyright and/or related rights. You are free to use this item in any way that is permitted by the copyright and related rights legislation that applies to your use. For other uses, you need to obtain permission from the rights-holder(s) directly, unless additional rights are indicated by a Creative Commons license in the record and/or on the work itself. For more information, please contact digitalcommons@fairfield.edu. 


\section{Beam-Helicity Asymmetries in Double-Charged-Pion Photoproduction on the Proton}

S. Strauch,,${ }^{1,32}$ B. L. Berman, ${ }^{1}$ G. Adams,${ }^{29}$ P. Ambrozewicz, ${ }^{10}$ M. Anghinolfi, ${ }^{15}$ B. Asavapibhop, ${ }^{21}$ G. Asryan, ${ }^{38}$ G. Audit, ${ }^{6}$ H. Avakian, ${ }^{14,33}$ H. Bagdasaryan, ${ }^{27}$ N. Baillie, ${ }^{37}$ J. P. Ball, ${ }^{2}$ N. A. Baltzell, ${ }^{32}$ S. Barrow, ${ }^{11}$ V. Batourine, ${ }^{19}$ M. Battaglieri, ${ }^{15}$ K. Beard, ${ }^{18}$ I. Bedlinskiy, ${ }^{17}$ M. Bektasoglu,${ }^{27,26}$ M. Bellis, ${ }^{4}$ N. Benmouna, ${ }^{1}$ C. Bennhold, ${ }^{1}$ A. S. Biselli, ${ }^{29,4}$

S. Boiarinov, ${ }^{17,33}$ S. Bouchigny, ${ }^{33,16}$ R. Bradford, ${ }^{4}$ D. Branford, ${ }^{9}$ W. J. Briscoe, ${ }^{1}$ W. K. Brooks, ${ }^{33}$ S. Bültmann,${ }^{27}$ V. D. Burkert, ${ }^{33}$ C. Butuceanu, ${ }^{37}$ J. R. Calarco, ${ }^{24}$ S. L. Careccia, ${ }^{27}$ D. S. Carman, ${ }^{26}$ B. Carnahan, ${ }^{5}$ S. Chen,${ }^{11}$ P. L. Cole,${ }^{33,13}$ A. Coleman, ${ }^{37}$ P. Coltharp,${ }^{11}$ D. Cords,${ }^{33, *}$ P. Corvisiero, ${ }^{15}$ D. Crabb,${ }^{36}$ H. Crannell, ${ }^{5}$ J. P. Cummings, ${ }^{29}$

P. V. Degtyarenko, ${ }^{33}$ H. Denizli, ${ }^{28}$ L. Dennis, ${ }^{11}$ E. De Sanctis, ${ }^{14}$ A. Deur, ${ }^{33}$ R. DeVita, ${ }^{15}$ K. V. Dharmawardane, ${ }^{27}$ K. S. Dhuga, ${ }^{1}$ C. Djalali, ${ }^{32}$ G. E. Dodge, ${ }^{27}$ J. Donnelly, ${ }^{12}$ D. Doughty, ${ }^{733}$ P. Dragovitsch, ${ }^{11}$ M. Dugger, ${ }^{2}$ S. Dytman, ${ }^{28}$ O. P. Dzyubak, ${ }^{32}$ H. Egiyan, ${ }^{37,33,24}$ K. S. Egiyan, ${ }^{38}$ L. Elouadrhiri, ${ }^{7,33}$ A. Empl, ${ }^{29}$ P. Eugenio, ${ }^{11}$ R. Fatemi, ${ }^{36}$ G. Fedotov, ${ }^{23}$ G. Feldman, ${ }^{1}$ R. J. Feuerbach, ${ }^{4}$ A. Fix ${ }^{22}$ T. A. Forest,${ }^{27}$ H. Funsten, ${ }^{37}$ G. Gavalian, ${ }^{38,27,24}$ G. P. Gilfoyle, ${ }^{31}$

K. L. Giovanetti, ${ }^{18}$ F. X. Girod, ${ }^{6}$ J. T. Goetz, ${ }^{3}$ R. W. Gothe, ${ }^{32}$ K. A. Griffioen, ${ }^{37}$ M. Guidal, ${ }^{16}$ N. Guler, ${ }^{27}$ L. Guo, ${ }^{33}$ V. Gyurjyan, ${ }^{33}$ C. Hadjidakis, ${ }^{16}$ R. S. Hakobyan, ${ }^{5}$ J. Hardie,,${ }^{73}$ D. Heddle, ${ }^{7,33}$ F. W. Hersman, ${ }^{24}$ K. Hicks, ${ }^{26}$ I. Hleiqawi, ${ }^{26}$ M. Holtrop, ${ }^{24}$ J. Hu, ${ }^{29}$ M. Huertas, ${ }^{32}$ C. E. Hyde-Wright, ${ }^{27}$ Y. Ilieva, ${ }^{1}$ D. G. Ireland, ${ }^{12}$ B. S. Ishkhanov, ${ }^{23}$ M. M. Ito, ${ }^{33}$ D. Jenkins, ${ }^{35}$ H. S. Jo, ${ }^{16}$ K. Joo, ${ }^{36,8}$ H. G. Juengst, ${ }^{1,27}$ J. D. Kellie, ${ }^{12}$ M. Khandaker, ${ }^{25}$ K. Y. Kim, ${ }^{28}$ K. Kim, ${ }^{19}$ W. Kim, ${ }^{19}$ A. Klein, ${ }^{27}$ F. J. Klein, ${ }^{33,5}$ A. V. Klimenko, ${ }^{27}$ M. Klusman, ${ }^{29}$ M. Kossov, ${ }^{17}$ L. H. Kramer, ${ }^{10,33}$ V. Kubarovsky, ${ }^{29}$ J. Kuhn, ${ }^{4}$ S. E. Kuhn, ${ }^{27}$ J. Lachniet, ${ }^{4}$ J. M. Laget,,${ }^{6,33}$ J. Langheinrich, ${ }^{32}$ D. Lawrence, ${ }^{21}$ T. Lee, ${ }^{24}$ A. C. S. Lima, ${ }^{1}$ K. Livingston, ${ }^{12}$ K. Lukashin, ${ }^{33,5}$ J. J. Manak, ${ }^{33}$ C. Marchand, ${ }^{6}$ S. McAleer, ${ }^{11}$ B. McKinnon, ${ }^{12}$ J. W. C. McNabb, ${ }^{4}$ B. A. Mecking, ${ }^{33}$ M. D. Mestayer,${ }^{33}$ C. A. Meyer, ${ }^{4}$ T. Mibe,${ }^{26}$ K. Mikhailov, ${ }^{17}$ R. Minehart, ${ }^{36}$ M. Mirazita, ${ }^{14}$ R. Miskimen, ${ }^{21}$ V. Mokeev,${ }^{33,23}$ S. A. Morrow, ${ }^{6}{ }^{16}$ V. Muccifora, ${ }^{14}$ J. Mueller, ${ }^{28}$ G. S. Mutchler,${ }^{30}$ P. Nadel-Turonski, ${ }^{1}$ J. Napolitano, ${ }^{29}$ R. Nasseripour, ${ }^{10,32}$ S. Niccolai, ${ }^{1,16}$ G. Niculescu, ${ }^{26,18}$ I. Niculescu, ${ }^{1,18}$ B. B. Niczyporuk, ${ }^{33}$ R. A. Niyazov,${ }^{27,33}$ M. Nozar, ${ }^{33}$ G. V. O’Rielly, ${ }^{1}$ M. Osipenko, ${ }^{15,23}$ A. I. Ostrovidov, ${ }^{11}$ K. Park, ${ }^{19}$ E. Pasyuk, ${ }^{2}$ C. Paterson, ${ }^{9}$ S. A. Philips, ${ }^{1}$ J. Pierce,${ }^{36}$ N. Pivnyuk,${ }^{17}$ D. Pocanic, ${ }^{36}$ O. Pogorelko, ${ }^{17}$ E. Polli,${ }^{14}$ S. Pozdniakov, ${ }^{17}$ B. M. Preedom,${ }^{32}$ J. W. Price,${ }^{3}$ Y. Prok,${ }^{33,20}$ D. Protopopescu, ${ }^{12}$ L. M. Qin, ${ }^{27}$ B. A. Raue, ${ }^{10,33}$ G. Riccardi, ${ }^{11}$ G. Ricco, ${ }^{15}$ M. Ripani, ${ }^{15}$ B. G. Ritchie, ${ }^{2}$ W. Roberts, ${ }^{27}$ F. Ronchetti, ${ }^{14}$ G. Rosner, ${ }^{12}$ P. Rossi, ${ }^{14}$ D. Rowntree, ${ }^{20}$ P. D. Rubin, ${ }^{31}$ F. Sabatié, ${ }^{6,27}$ C. Salgado, ${ }^{25}$ J. P. Santoro,, 35 V. Sapunenko, ${ }^{15,33}$ R. A. Schumacher, ${ }^{4}$ V. S. Serov, ${ }^{17}$ A. Shafi, ${ }^{1}$ Y. G. Sharabian, ${ }^{28,33}$ J. Shaw, ${ }^{21}$ A. V. Skabelin, ${ }^{20}$ E. S. Smith, ${ }^{33}$ L. C. Smith, ${ }^{36}$ D. I. Sober, ${ }^{5}$ A. Stavinsky, ${ }^{17}$ S. S. Stepanyan, ${ }^{19}$ S. Stepanyan, ${ }^{33,38}$ B. E. Stokes,${ }^{11}$ P. Stoler ${ }^{29}$ I. I. Strakovsky, ${ }^{1}$ R. Suleiman, ${ }^{20}$ M. Taiuti, ${ }^{15}$ S. Taylor,${ }^{30,26}$ D. J. Tedeschi, ${ }^{32}$ U. Thoma,${ }^{33, \dagger}$ R. Thompson, ${ }^{28}$ A. Tkabladze,${ }^{26,1}$ S. Tkachenko, ${ }^{27}$ L. Todor, ${ }^{31}$ C. Tur, ${ }^{32}$ M. Ungaro, ${ }^{29,8}$ M. F. Vineyard,${ }^{34,31}$ A. V. Vlassov, ${ }^{17}$ K. Wang, ${ }^{36}$ L. B. Weinstein, ${ }^{27}$ D. P. Weygand, ${ }^{33}$ M. Williams, ${ }^{4}$ E. Wolin, ${ }^{33}$ M. H. Wood, ${ }^{32,21}$ A. Yegneswaran, ${ }^{33}$ J. Yun, ${ }^{27}$ L. Zana, ${ }^{24}$ and J. Zhang ${ }^{27}$

\section{(CLAS Collaboration)}

\footnotetext{
${ }^{1}$ The George Washington University, Washington, District of Columbia 20052, USA

${ }^{2}$ Arizona State University, Tempe, Arizona 85287-1504, USA

${ }^{3}$ University of California at Los Angeles, Los Angeles, California 90095-1547, USA

${ }^{4}$ Carnegie Mellon University, Pittsburgh, Pennsylvania 15213, USA

${ }^{5}$ Catholic University of America, Washington, District of Columbia 20064, USA

${ }^{6}$ Service de Physique Nucléaire, CEA-Saclay, F91191 Gif-sur-Yvette,Cedex, France

${ }^{7}$ Christopher Newport University, Newport News, Virginia 23606, USA

${ }^{8}$ University of Connecticut, Storrs, Connecticut 06269, USA

${ }^{9}$ Edinburgh University, Edinburgh EH9 3JZ, United Kingdom

${ }^{10}$ Florida International University, Miami, Florida 33199, USA

${ }^{11}$ Florida State University, Tallahassee, Florida 32306, USA

${ }^{12}$ University of Glasgow, Glasgow G12 8QQ, United Kingdom

${ }^{13}$ Idaho State University, Pocatello, Idaho 83209, USA

${ }^{14}$ Laboratori Nazionali di Frascati, INFN, Frascati, Italy

${ }^{15}$ Sezione di Genova, INFN, 16146 Genova, Italy

${ }^{16}$ Institut de Physique Nucleaire ORSAY, Orsay, France

${ }^{17}$ Institute of Theoretical and Experimental Physics, Moscow, 117259, Russia

${ }^{18}$ James Madison University, Harrisonburg, Virginia 22807, USA

${ }^{19}$ Kyungpook National University, Daegu 702-701, South Korea

${ }^{20}$ Massachusetts Institute of Technology, Cambridge, Massachusetts 02139-4307, USA
} 


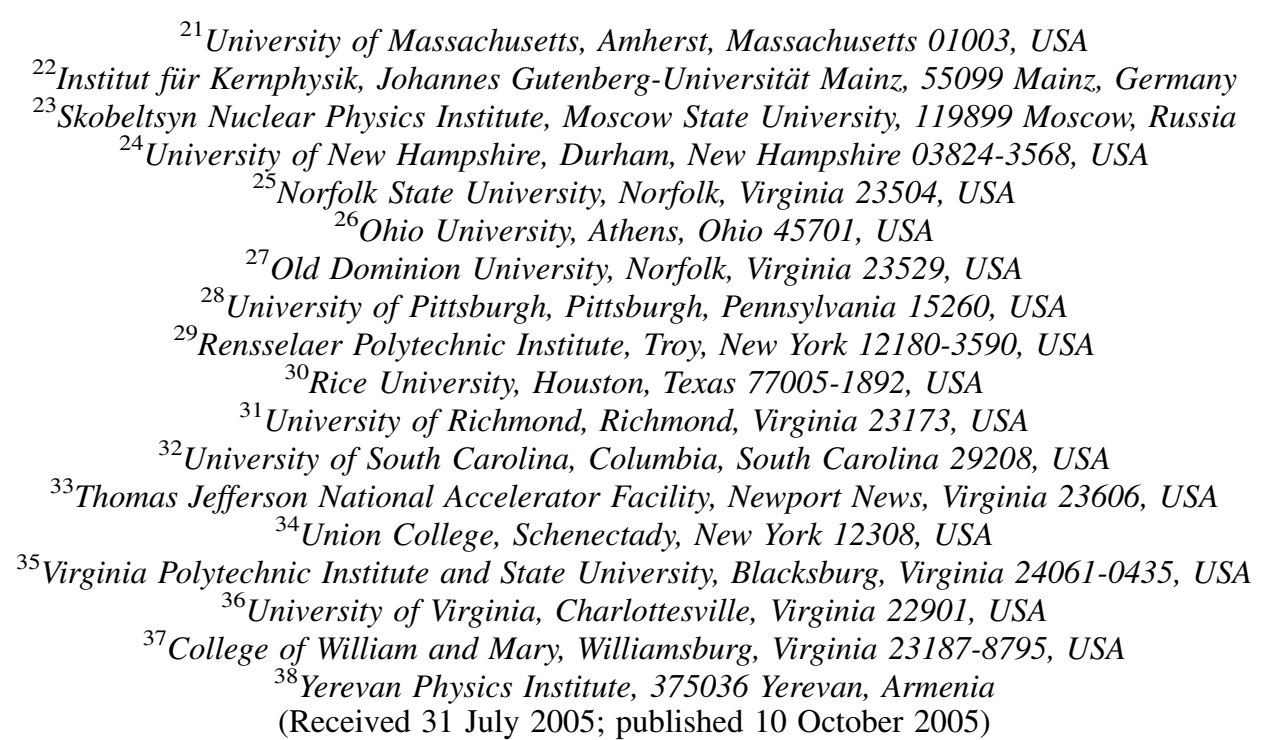

Beam-helicity asymmetries for the two-pion-photoproduction reaction $\vec{\gamma} p \rightarrow p \pi^{+} \pi^{-}$have been studied for the first time in the resonance region for center-of-mass energies between 1.35 and $2.30 \mathrm{GeV}$. The experiment was performed at Jefferson Lab with the CEBAF Large Acceptance Spectrometer using circularly polarized tagged photons incident on an unpolarized hydrogen target. Beam-helicity-dependent angular distributions of the final-state particles were measured. The large crosssection asymmetries exhibit strong sensitivity to the kinematics and dynamics of the reaction. The data are compared with the results of various phenomenological model calculations, and show that these models currently do not provide an adequate description for the behavior of this new observable.

The study of the baryon spectrum provides an avenue to a deeper understanding of the strong interaction, since the properties of the excited states of baryons reflect the dynamics and relevant degrees of freedom within them. Many nucleon resonances in the mass region above $1.6 \mathrm{GeV}$ decay predominantly through either $\pi \Delta$ or $\rho N$ intermediate states into $\pi \pi N$ final states (see the Particle Data Group review [1]). Resonances predicted by symmetric quark models, but not observed in the $\pi N$ channel (the so-called "missing" resonances), are predicted to lie in the region of $W>1.8 \mathrm{GeV}$ [2]. This makes electromagnetic double-pion production an important tool in the investigation of the structure of the nucleon.

To date, a rather large amount of unpolarized crosssection measurements of double-pion photoproduction and electroproduction on the proton have been reported by several collaborations [3-12]. However, the database collected for polarization observables remains quite sparse. Polarization degrees of freedom in charged double-pion production have been studied at SLAC [13] and in the context of the Gerasimov-Drell-Hearn sum rule at MAMI [14].

On the theoretical side, some experience has been gained during the last decade [15-23]. It should be noted that the various models which are presently used are constructed according to the same scheme-effective Lagrangian densities, where the parameters for resonant and background mechanisms (contact and $u, t$-channel pole terms) are either taken from other experiments or are treated as free parameters in the analysis. Aside from the wide variations in the corresponding coupling constants allowed by the Particle Data Group listing, the primary source of differences between the models is the treatment of the background, which appears to be very complicated in the effective Lagrangian approach for double-pion photoproduction. A better understanding of the double-pion photoproduction dynamics is vital for the reliable extraction of $N^{*}$ photocouplings. Polarization data, being particularly sensitive to interference effects, are expected to provide valuable constraints.

In this Letter, we report the first comprehensive measurement of the beam-helicity asymmetry [24]

$$
I^{\odot}=\frac{1}{P_{\gamma}} \frac{\sigma^{+}-\sigma^{-}}{\sigma^{+}+\sigma^{-}}
$$

in the $\vec{\gamma} p \rightarrow p \pi^{+} \pi^{-}$reaction, for energies $W$ between 1.35 and $2.30 \mathrm{GeV}$ in the center of mass, where the photon beam is circularly polarized and neither target nor recoil polarization is specified. $P_{\gamma}$ is the degree of circular polarization of the photon and $\sigma^{ \pm}$are the cross sections for the two photon-helicity states $\lambda_{\gamma}= \pm 1$. Here, we give a brief overview of our data and demonstrate, by means of a phenomenological model, the sensitivity of this observable to the dynamics of the reaction. 
The experiment was performed in Hall B at the Thomas Jefferson National Accelerator Facility (Jefferson Lab). Longitudinally polarized electrons with an energy $E_{0}=$ $2.445 \mathrm{GeV}$ were incident on a thin radiator. The beam polarization was routinely monitored during data taking by a Møller polarimeter and was, on average, 0.67. A photon tagger system [25] was used to tag photons in the energy range between 0.5 and $2.3 \mathrm{GeV}$, with an energy resolution of $0.1 \% E_{0}$. The degree of circular polarization of the photon beam is proportional to the electron-beam polarization and is a monotonic function of the ratio of the photon and incident electron energies [26]. The degree of photon-beam polarization varied from $\approx 0.16$ at the lowest photon energy up to $\approx 0.66$ at the highest energy. The photon-helicity state changes with the electron-beam helicity, which was flipped pseudorandomly at a rate of $30 \mathrm{~Hz}$. The collimated photon beam irradiated an $18 \mathrm{~cm}$ thick liquid-hydrogen target. The final-state hadrons were detected in the CEBAF Large Acceptance Spectrometer (CLAS) [27]. The CLAS provides a large coverage for charged particles that includes particle momenta down to $0.25 \mathrm{GeV} / c$ and polar angles in the range $8^{\circ}<\theta_{\text {lab }}<$ $145^{\circ}$. The event trigger required a coincidence between a scattered-electron signal from the photon tagger and at least one charged track in the CLAS. The four-momentum vectors of the particles were reconstructed from their tracks in the toroidal magnetic field of the spectrometer by a set of three drift-chamber packages and by particle identification using time-of-flight information from plastic scintillators located about $5 \mathrm{~m}$ from the target.

The $\vec{\gamma} p \rightarrow p \pi^{+} \pi^{-}$reaction channel was identified in this kinematically complete experiment by the missingmass technique, requiring either the detection of all three final-state particles or the detection of two out of the three particles.

A schematic view of the reaction, together with angle definitions, is shown in Fig. 1. A total of $3 \times 10^{7} p \pi^{+} \pi^{-}$

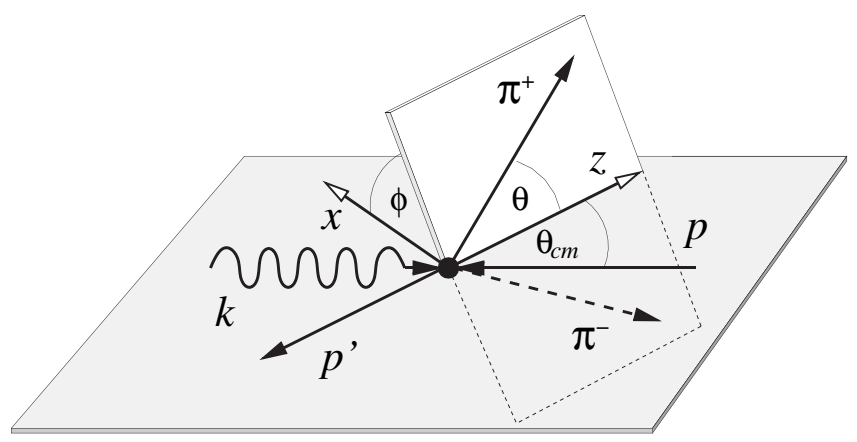

FIG. 1. Angle definitions for the circularly polarized realphoton reaction $\vec{\gamma} p \rightarrow p \pi^{+} \pi^{-} ; \theta_{\mathrm{cm}}$ is defined in the overall center-of-mass frame, and $\theta$ and $\phi$ are defined as the $\pi^{+}$polar and azimuthal angles in the rest frame of the $\pi^{+} \pi^{-}$system with the $z$ direction along the total momentum of the $\pi^{+} \pi^{-}$system (helicity frame). events were accumulated for both helicity states $N^{ \pm}$. Experimental values of the helicity asymmetry were then obtained as

$$
I_{\exp }^{\odot}=\frac{1}{\bar{P}_{\gamma}} \frac{N^{+} / \alpha^{+}-N^{-} / \alpha^{-}}{N^{+} / \alpha^{+}+N^{-} / \alpha^{-}}
$$

where $\alpha^{ \pm}=\frac{1}{2}\left(1 \pm a_{c}\right)$ accounts for helicity-dependent differences in the luminosity due to a small electronbeam-charge asymmetry $a_{c} \approx-0.0044$. The value of $a_{c}$ was determined from helicity asymmetries in single-pion photoproduction for data that were obtained simultaneously with the double-pion photoproduction data. Any observed asymmetry in this reaction is instrumental [28]. The experimental asymmetries have not been corrected for the CLAS acceptance. In order to allow for an analysis as model independent as possible, the data are compared with event-weighted mean values of asymmetries from model calculations [29]. The determination of these mean values

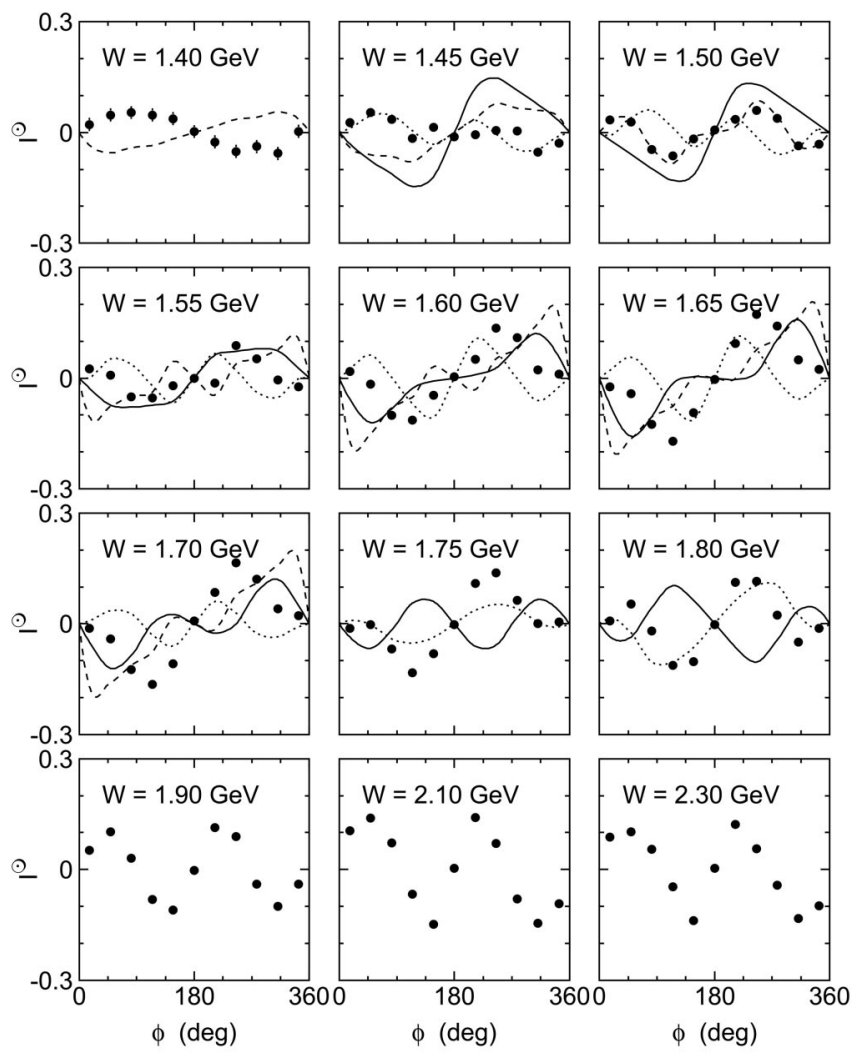

FIG. 2. Angular distributions for selected center-of-mass energy bins (each with $\Delta W=50 \mathrm{MeV}$ ) of the cross-section asymmetry for the $\vec{\gamma} p \rightarrow p \pi^{+} \pi^{-}$reaction. The data are integrated over the detector acceptance. The statistical uncertainties are mostly smaller than the symbol size. The solid and dotted curves are the results from model calculations by Mokeev et al. [19-21] (for $1.45 \mathrm{GeV} \leq W \leq 1.80 \mathrm{GeV}$ ) with relative phases of 0 and $\pi$ between the background- and $\pi \Delta$-subchannel amplitudes, respectively. The dashed curves show results of calculations by Fix and Arenhövel [23] (for $W \leq 1.70 \mathrm{GeV}$ ). 
does not require a knowledge of the CLAS acceptance. The only major source of systematic uncertainty is the value for the beam polarization, which is known to about $3 \%$. The uncertainty from the beam-charge asymmetry is negligible (less than $10^{-3}$ ).

Figure 2 shows $\phi$ angular distributions of the helicity asymmetry for various selected $50 \mathrm{MeV}$-wide center-ofmass energy bins between $W=1.40$ and $2.30 \mathrm{GeV}$.

The data are integrated over the full CLAS acceptance. The analysis shows large asymmetries which change markedly with $W$ up to $1.80 \mathrm{GeV}$; thereafter they remain rather stable. The asymmetries are odd functions of $\phi$ and vanish for coplanar kinematics $\left(\phi=0\right.$ and $\left.180^{\circ}\right)$, as expected from parity conservation [24]. The large number of observed $\vec{\gamma} p \rightarrow p \pi^{+} \pi^{-}$events allows for a confident analysis of the data in selected kinematic regions, making it possible to tune the different parts of the production amplitude independently. An example of distributions which are more differential than those of Fig. 2 is given in Fig. 3. The data at $W=1.50 \mathrm{GeV}$ are divided into nine bins in the invariant mass $M\left(p \pi^{+}\right)$.

The data in Figs. 2 and 3 are compared with results of available phenomenological models. In the approach by Mokeev et al. (solid curves), double-charged-pion photoproduction and electroproduction are described by a set of quasi-two-body mechanisms with unstable particles in the intermediate states: $\pi \Delta, \rho N, \pi N(1520), \pi N(1680)$, and $\pi \Delta(1600)$ and with subsequent decays to the $\pi^{+} \pi^{-} p$ final state [19-21]. Residual direct $\pi^{+} \pi^{-} p$ mechanisms are parametrized by exchange diagrams [21]. The first two quasi-two-body channels mentioned above are described by a coherent sum of $s$-channel $N^{*}$ contributions and nonresonant mechanisms [19]. All well established resonances
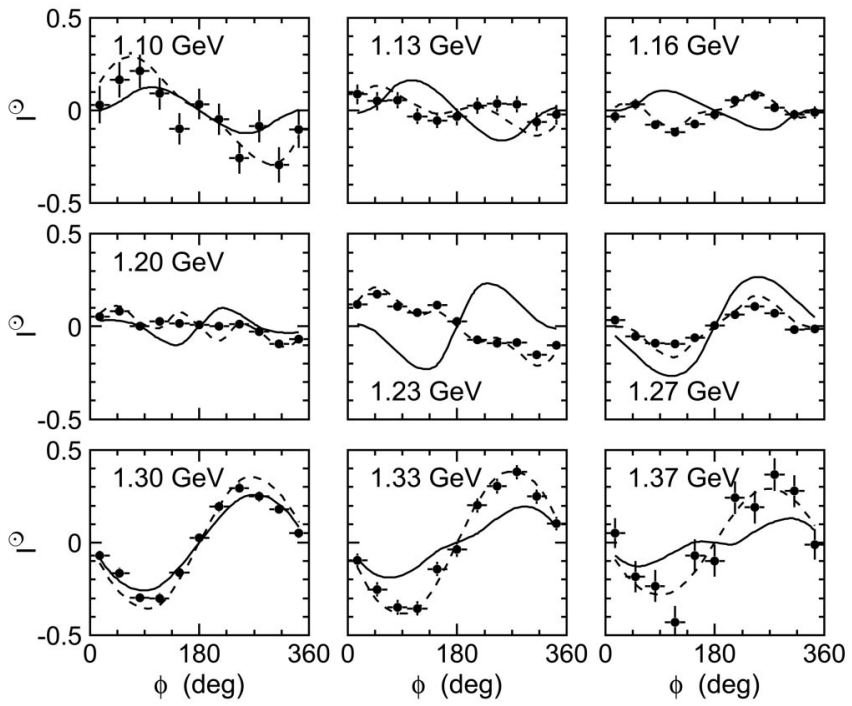

FIG. 3. Helicity asymmetries at $W=1.50 \mathrm{GeV}$ for nine bins of the invariant mass $M\left(p \pi^{+}\right)$, as indicated. The solid curves are the results of Mokeev et al. [19-21]. The dashed curves show results of calculations by Fix and Arenhövel [23]. with observed double-pion decays are included, plus $\Delta(1600), N(1700), N(1710)$, and a new state, $N(1720)$ with $J^{\pi}=3 / 2^{+}$, possibly observed in CLAS doublepion data [9]. $N^{*}$ and nonresonant parameters are fitted to the CLAS cross-section data for virtual-photon doublecharged-pion production [9]. The model provides a good description of all available CLAS cross-section and world data on double-pion photoproduction and electroproduction at $W<1.9 \mathrm{GeV}$ and $Q^{2}<1.5 \mathrm{GeV}^{2}$.

Results also have been obtained by Fix and Arenhövel using the model described in [23]. They use an effective Lagrangian approach with Born and resonance diagrams at the tree level. The model includes the nucleon, the $\Delta(1232), \quad N(1440), \quad N(1520), \quad N(1535), \quad N(1680)$, $\Delta(1620), N(1675)$, and $N(1720)$ resonances, as well as the $\sigma$ and $\rho$ mesons. The corresponding results are shown in Figs. 2 and 3 as dashed curves. For completeness, we note that the recent work of Roca [18] shows our preliminary data [30] in the framework of the Valencia model for double-pion photoproduction.

Although both models had previously provided a good description of unpolarized cross sections, neither of the models is able to provide a reasonable description of the beam-asymmetry data over the entire kinematic range covered in this experiment. Even though the model predictions agree remarkably well for certain conditions (see, e.g., the dashed curves in Fig. 3), for other conditions they are much worse and sometimes even out of phase entirely.

As is noted above, the main theoretical challenge for double-pion photoproduction lies in the fact that several subprocesses may contribute, even though any given individual contribution may be small. In this connection, the polarization measurements should be very helpful in separating the individual terms. The particular sensitivity of the beam asymmetry to interference effects among various amplitudes is illustrated in Fig. 2. The dotted curves show results of calculations by Mokeev et al. with a relative phase of $\pi$ between the background- and $\pi \Delta$-subchannel amplitudes. The access to interference effects permit a cleaner separation of background and resonances. This in turn makes it possible to make more reliable statements about the existence and properties of nucleon resonances.

Figure 4 shows the helicity asymmetry as a function of the invariant mass $M\left(p \pi^{-}\right)$for two different values of $W$ and a fixed value of $\phi$.

This is a typical case. The most interesting features of these data are the changes that occur as $M\left(p \pi^{-}\right)$traverses the $\Delta(1232)$ resonance. At $W=1.55 \mathrm{GeV}$, a maximum is seen in the region of this resonance. We see a similar trend in the region of the higher-mass resonances around $1.60 \mathrm{GeV}$ for $W=1.95 \mathrm{GeV}$. This hints at the way in which the helicity asymmetry (along with other polarization observables) could be used in studies of baryon spectroscopy. Of particular interest is the study of sequential decays of resonances, such as $N(1520) \rightarrow \pi \Delta \rightarrow \pi \pi N$, or 


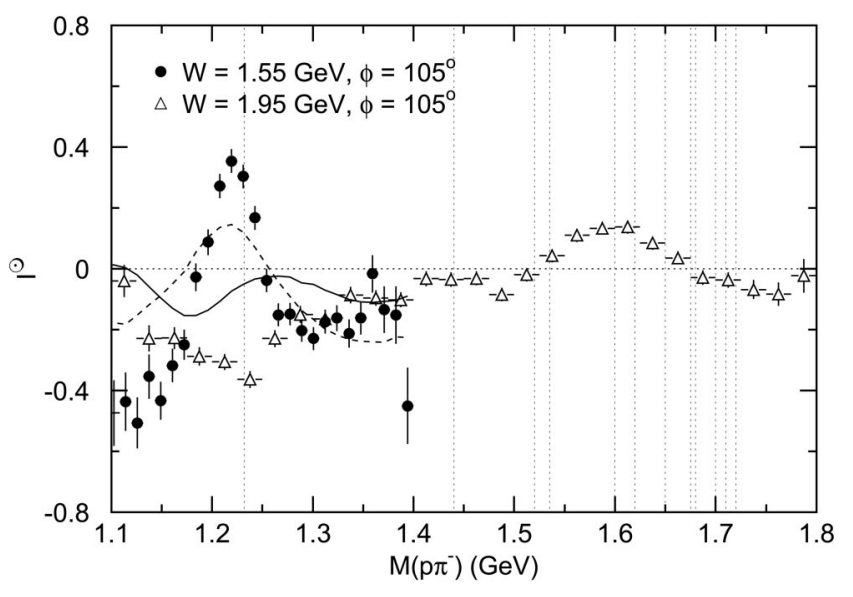

FIG. 4. Helicity asymmetry as a function of the invariant mass $M\left(p \pi^{-}\right)$for $W=1.55 \mathrm{GeV}$ (filled circles) and $1.95 \mathrm{GeV}$ (open triangles) and a $30^{\circ}$-wide $\phi$-angle range centered at $\phi=105^{\circ}$. The curves are the results of Mokeev et al. [19-21] (solid line) and Fix and Arenhövel [23] (dashed line) for $W=1.55 \mathrm{GeV}$ only. Note that the result of Fix and Arenhövel is in phase with the data (filled circles) and that of Mokeev et al. is not. The vertical lines indicate the masses of the known $N$ and $\Delta$ resonances.

$N(1700) \rightarrow \pi N(1520) \rightarrow \pi \pi N$, which can be studied at moderate values of $W$ from 1.5 to $1.9 \mathrm{GeV}$; see Ref. [10]. Here, the $\rho$-production channel is also open. This is the energy range where yet-unobserved resonances are predicted to lie [2].

In summary, we have given a brief overview of our $\vec{\gamma} p \rightarrow p \pi^{+} \pi^{-}$data, and we have demonstrated, by means of phenomenological models, the sensitivity of this helicity-asymmetry observable to the dynamics of the reaction. The large amount of high-quality data that we have obtained opens the path for a series of further investigations. Obvious next steps are (1) a better theoretical understanding of the reaction and (2) an attempt to describe simultaneously our polarized double-charged pion photoproduction data and other CLAS data obtained with unpolarized real [11] and virtual [9] photons.

We see, even from the small sample of data shown here, that existing theoretical models have severe shortcomings in the description of the beam-helicity asymmetries. In the region of overlapping nucleon resonances (and uncontrolled backgrounds), it clearly will be a challenge to any theoretical model to describe this new observable that depends so sensitively on the interferences between them. Yet, without a proper understanding of the $\pi \pi N$ channel the problem of the missing resonances is unlikely to be resolved.

We would like to thank the staff of the Accelerator and Physics Divisions at Jefferson Lab, as well as the Italian Istituto Nazionale di Fisica Nucleare, the French Centre National de la Recherche Scientifique and Commissariat à l'Energie Atomique, the U.S. Department of Energy and
National Science Foundation, and the Korea Science and Engineering Foundation. Southeastern Universities Research Association (SURA) operates the Thomas Jefferson National Accelerator Facility under U.S. Department of Energy contract No. DE-AC0584ER40150. The GWU Experimental Nuclear Physics Group is supported by the U.S. Department of Energy under Grant No. DE-FG02-95ER40901.

*Deceased.

${ }^{\dagger}$ Current address: Physikalisches Institut der Universität Gießen, 35392 Gießen, Germany.

[1] S. Eidelman et al., Phys. Lett. B 592, 1 (2004).

[2] S. Capstick and W. Roberts, Phys. Rev. D 49, 4570 (1994).

[3] ABBHHM Collaboration, Phys. Rev. 175, 1669 (1968).

[4] ABBHHM Collaboration, Phys. Rev. 188, 2060 (1969).

[5] A. Braghieri et al., Phys. Lett. B 363, 46 (1995).

[6] F. Härter et al., Phys. Lett. B 401, 229 (1997).

[7] M. Wolf et al., Eur. Phys. J. A 9, 5 (2000).

[8] Y. Assafiri et al., Phys. Rev. Lett. 90, 222001 (2003).

[9] M. Ripani et al., Phys. Rev. Lett. 91, 022002 (2003).

[10] S.A. Philips, Ph.D. thesis, The George Washington University, 2003.

[11] M. Bellis, Ph.D. thesis, Rensselaer Polytechnic Institute, 2003.

[12] W. Langgärtner et al., Phys. Rev. Lett. 87, 052001 (2001).

[13] J. Ballam et al., Phys. Rev. D 5, 545 (1972).

[14] J. Ahrens et al. (GDH and A2 Collaborations), Phys. Lett. B 551, 49 (2003).

[15] J. A. Gomez-Tejedor and E. Oset, Nucl. Phys. A600, 413 (1996).

[16] J. C. Nacher et al., Nucl. Phys. A695, 295 (2001).

[17] J. C. Nacher and E. Oset, Nucl. Phys. A697, 372 (2002).

[18] L. Roca, Nucl. Phys. A748, 192 (2005).

[19] V. I. Mokeev et al., Yad. Fiz. 64, 1368 (2001); [Phys. At. Nucl. 64, 1292 (2001)].

[20] V. Burkert et al., Proceedings of Few-Body 17 (Elsevier, Amsterdam, 2004), p. S231.

[21] V. I. Mokeev et al., Proceedings of NSTAR2004 (World Scientific, New Jersey, 2004), p. 321.

[22] W. Roberts and A. Rakotovao, hep-ph/9708236.

[23] A. Fix and H. Arenhövel, nucl-th/0503042.

[24] W. Roberts and T. Oed, Phys. Rev. C 71, 055201 (2005).

[25] D. I. Sober et al., Nucl. Instrum. Methods Phys. Res., Sect. A 440, 263 (2000).

[26] H. Olsen and L. C. Maximon, Phys. Rev. 114, 887 (1959).

[27] B. A. Mecking et al., Nucl. Instrum. Methods Phys. Res., Sect. A 503, 513 (2003).

[28] I. S. Barker, A. Donnachie, and J. K. Storrow, Nucl. Phys. B95, 347 (1975).

[29] The mean value $\bar{I}^{\odot}$ of model asymmetries in a kinematical bin is given by $\bar{P}_{\gamma} \bar{I}^{\odot}=\frac{1}{N} \sum_{i} P_{\gamma, i} I_{i}^{\odot}$, where the sum runs over all $N$ events observed in that bin; $P_{\gamma, i}$ is the calculated beam polarization at the corresponding $W$ and $I_{i}^{\odot}$ is the model asymmetry for the kinematics of each of those events.

[30] S. Strauch et al., Proceedings of NSTAR2004 (World Scientific, New Jersey, 2004), p. 317. 